\title{
The Pain of Sleep Loss: A Brain Characterization in Humans
}

\author{
๑DAdam J. Krause, ${ }^{1}$ @Aric A. Prather, ${ }^{2}$ Tor D. Wager, ${ }^{3}$ Martin A. Lindquist, ${ }^{4}$ and Matthew P. Walker ${ }^{1,5}$ \\ ${ }^{1}$ Center for Human Sleep Science, Department of Psychology, University of California, Berkeley, California 94720-1650, ${ }^{2}$ Department of Psychiatry, \\ University of California, San Francisco, California 94103, ${ }^{3}$ Department of Psychology and Neuroscience, University of Colorado, Boulder, Colorado 80302, \\ ${ }^{4}$ Department of Biostatistics, Johns Hopkins University, Baltimore, Maryland 21205, and ${ }^{5}$ Helen Wills Neuroscience Institute, University of California, \\ Berkeley, California 94720-1650
}

Sleep loss increases the experience of pain. However, the brain mechanisms underlying altered pain processing following sleep deprivation are unknown. Moreover, it remains unclear whether ecologically modest night-to-night changes in sleep, within an individual, confer consequential day-to-day changes in experienced pain. Here, we demonstrate that acute sleep deprivation amplifies pain reactivity within human (male and female) primary somatosensory cortex yet blunts pain reactivity in higher-order valuation and decision-making regions of the striatum and insula cortex. Consistent with this altered neural signature, we further show that sleep deprivation expands the temperature range for classifying a stimulus as painful, specifically through a lowering of pain thresholds. Moreover, the degree of amplified reactivity within somatosensory cortex following sleep deprivation significantly predicts this expansion of experienced pain across individuals. Finally, outside of the laboratory setting, we similarly show that even modest nightly changes in sleep quality (increases and decreases) within an individual determine consequential day-to-day changes in experienced pain (decreases and increases, respectively). Together, these data provide a novel framework underlying the impact of sleep loss on pain and, furthermore, establish that the association between sleep and pain is expressed in a night-to-day, bidirectional relationship within a sample of the general population. More broadly, our findings highlight sleep as a novel therapeutic target for pain management within and outside the clinic, including circumstances where sleep is frequently short yet pain is abundant (e.g., the hospital setting).

Key words: fMRI; pain; sleep deprivation

Significance Statement

Are you experiencing pain? Did you have a bad night of sleep? This study provides underlying brain and behavioral mechanisms explaining this common co-occurrence. We show that sleep deprivation enhances pain responsivity within the primary sensing regions of the brain's cortex yet blunts activity in other regions that modulate pain processing, the striatum and insula. We further establish that even subtle night-to-night changes in sleep in a sample of the general population predict consequential day-to-day changes in pain (bidirectionally). Considering the societal rise in chronic pain conditions in lock-step with the decline in sleep time through the industrial world, our data support the hypothesis that these two trends may not simply be co-occurring but are significantly interrelated.

\section{Introduction}

Sleep loss amplifies the experience of pain. Causal manipulations in animals demonstrate that sleep deprivation enhances nociceptive behavior (Lautenbacher et al., 2006). Experiments in humans establish that total (Schuh-Hofer et al., 2013), partial (Faraut et al., 2015), and selective sleep deprivation (Lentz et al.,

Received Sept. 17, 2018; revised Dec. 6, 2018; accepted Dec. 7, 2018.

Author contributions: A.J.K. wrote the first draft of the paper; A.J.K., A.A.P., T.D.W., M.A.L., and M.P.W. edited the paper; A.J.K. designed research; A.J.K. performed research; A.J.K. analyzed data; A.J.K. and M.P.W. wrote the paper.

We thank the following research assistants: Samika Kumar, Harlene Grewel, Grace Kim, Kaycee Ching, Harshika Chowdhary, Jessica Kendall-Bar, Mandana Mostofi, and Jay Gupta.

The authors declare no competing financial interests.

Correspondence should be addressed to Matthew P. Walker at mpwalker@berkeley.edu.

https://doi.org/10.1523/JNEUROSCI.2408-18.2018

Copyright $\odot 2019$ the authors $\quad 0270-6474 / 19 / 392291-10 \$ 15.00 / 0$
1999; Roehrs et al., 2006) increase pain, including a lowering of pain thresholds (Schrimpf et al., 2015).

Despite these robust links, the central brain mechanisms underlying the impact of sleep loss on pain perception remain unknown in, any species. Beyond gross manipulations of sleep within the laboratory, it is similarly unknown whether modest, societally common, night-to-night changes in sleep within an individual confer consequential day-to-day changes in experienced pain. This study sought to address both of these currently unanswered questions.

Although no reports have explored the brain basis of sleep loss-induced hyperalgesia, neuroimaging studies in non-sleepdeprived individuals have established a pattern of functional magnetic resonance imaging (fMRI) activity in regions respon- 
sive to pain (Brown et al., 2011; Wager et al., 2013; for review, see Tracey and Mantyh, 2007; Woo et al., 2017). These include primary and secondary somatosensory cortices that map noxious stimuli (Apkarian et al., 2005; Atlas et al., 2014). Activity within somatosensory cortex scales as a function of pain intensity, including at pain threshold (Coghill et al., 1999; Timmermann et al., 2001; Apkarian et al., 2005; Atlas et al., 2014).

Corticolimbic regions of the cingulate and insula are consistently activated by pain and are involved in the second-order mapping of internal state changes associated with pain (Craig, 2002). Subcortical regions, including spinal-afferent-receiving regions of the thalamus, as well as nucleus accumbens (NAcc), also show robust pain reactivity (Navratilova and Porreca, 2014). The NAcc in particular plays a role in encoding affective value and saliency, including that of pain (Redgrave et al., 2008; Schultz, 2013; Navratilova and Porreca, 2014), allowing the modulation of ongoing pain through descending brain and spinal cord pathways (Navratilova and Porreca, 2014).

This pain-reactive network provides a set of candidatehypothesized ROIs to account for increased pain caused by sleep deprivation. Specifically, activity within regions related to the magnitude of experienced pain, including somatosensory cortex and thalamus should scale with the degree of enhanced pain following sleep loss. In contrast, higher-order evaluative regions of the insula, cingulate, and NAcc that play a role in the gating, integration, and relief from classification of pain signals, would conversely fail to appropriately modulate pain following sleep loss, evidenced by a reduction in activity.

Beyond changes in functional brain network activity associated with acute sleep deprivation, an additionally unanswered question centers on whether more subtle changes in sleep from one night to the next, within an individual, lead to ensuing daily fluctuations in pain sensitivity. This not only represents an extended test of the central sleep-pain hypothesis, complementing experimental acute sleep deprivation manipulations, but further determines whether such findings hold public health relevance. Previous survey studies have established that sleep disruption and pain are significantly linked (Afolalu et al., 2018). However, these studies use cross-sectional analyses at a single time point, and in clinical populations (e.g., chronic pain and/or insomnia) (Tang et al., 2012). It therefore remains unknown whether microlongitudinal studies that examine changes in night-to-night sleep and day-to-day pain within an individual are significantly and predictively interrelated. Moreover, which features of sleep (e.g., duration, quality, efficiency) are the deterministic factors underlying the sleep-pain relationship remains similarly unknown (Edwards et al., 2008).

Building on this evidence, we test a set of interrelated hypotheses: (1) Acute sleep deprivation increases pain-related activity within the human primary somatosensory cortex and subcortical thalamus yet disrupts pain processes in regions of second order limbic cortex and pain relief-associated regions of the NAcc. Moreover, the magnitude of such changes will predict the lowering in thermal pain thresholds and thus increased range of pain sensation following sleep deprivation. (2) Outside of the laboratory, we additionally tested whether ecologically typical nightly changes of sleep within individuals in a sample of the general population result in a similar lowering of pain thresholds, leading to daily increases in experienced pain.

\section{Materials and Methods}

\section{Experimental design}

To test the experimental hypotheses, two related studies were performed: (1) an in-laboratory study and (2) an online study. The in-laboratory study involved 25 healthy adult participants enrolled in a counterbalanced, repeated-measures design involving two conditions: one night of sleep and one night of sleep deprivation (Fig. 1A). In each condition, participants performed a standardized thermal pain sensitivity assessment. Thermal pain thresholds were assessed outside of the fMRI scanner, with additional pain-experiential questions asked during the task inside the scanner (both described below).

In the sleep-deprived session, participants arrived at the laboratory at 10:00 P.M. and were continuously monitored throughout the enforced waking period. Activities during the sleep deprivation period were limited to use of internet, Email, short walks, reading, movies of low emotionality, and playing board games, thereby providing a standardized regimen of waking activity without undue stress. In the sleep-rested session, participants came to the laboratory at 8:00 P.M. and were prepared for an $\sim 8 \mathrm{~h}$ in-bed polysomnographic night of sleep recording in the laboratory (11:00 P.M. to 7:30 A.M. $\pm 30 \mathrm{~min}$; details below). The following morning at $\sim 8: 30$ A.M. ( $\pm 60 \mathrm{~min}$ ), participants underwent quantitative sensory testing outside of the MRI scanner to determine their pain threshold (described in detail below). Thereafter, participants performed a thermal pain sensitivity task in the fMRI scanner. These two sessions were separated by at least $7 \mathrm{~d}$, with the order of the sleep-rested and deprived sessions counterbalanced across subjects.

In the online phase of the study, a separate cohort of 236 users of Amazon Mechanical Turk (MTurk) were assessed across 2 nights and 2 subsequent days, sleeping as they chose (Fig. $1 B$, described below). This allowed for a test of how natural night-to-night variability in sleep quality, efficiency, and duration were associated with consequential day-today changes in pain.

Studies were approved by the local human ethics committee, with all participants providing written informed consent.

Participants: in-laboratory study. Twenty-five healthy adults, age $18-30$ years (mean \pm SD: $20.8 \pm 1.95$ years, $48 \%$ female) completed a repeated-measures crossover design (described above). Participants abstained from caffeine and alcohol for the $72 \mathrm{~h}$ before and during the entire course of the study and kept a normal sleep-wake rhythm (7-9 h of sleep per night with sleep onset before 1:00 A.M. in the morning and rise time no later than 9:00 A.M.) for the 3 nights before the study participation, as verified by sleep logs and actigraphy. 
Exclusion criteria, assessed using a prescreening semistructured interview, were as follows: a history of sleep disorders, chronic pain or pain disorder, current pain or injuries, neurologic disorders, autoimmune disorders, open and closed head injury, Axis I psychiatric disorders according to the DSM-IV-TR criteria (encompassing mental disorders, including depression, anxiety disorders, bipolar disorder, attention deficit disorder, and schizophrenia), history of drug abuse, and current use of antidepressant, psychostimulant, or hypnotic medication. Subjects who reported drinking 3 or more caffeine-containing beverages a day, such as caffeinated coffee, tea, or soft drinks, were excluded.

Finally, before their first session, participants underwent quantitative sensory testing to measure baseline thermal sensitivity. Two temperature levels were established for each participant to be used in subsequent fMRI pain tasks in both conditions: first, a lower temperature described as "warm, but not painful"; and, second, a higher temperature consistently described as "painfully hot" (corresponding to a 7/10 on a visual analog pain scale). These individualized temperatures were estimated using established psychophysical methods (described below). Crucially, by matching temperatures across experimental conditions, further comparisons of brain pain reactivity controlled for objective stimulus temperature. This importantly means that observed neural changes caused by sleep deprivation could not be due to subjective differences in pain experience elicited by objectively different stimulus temperatures.

To assess the degree of difference between the structured sleep schedule of the experiment and each participant's unrestricted sleep schedule, participants completed the Pittsburgh Sleep Quality Index upon study entry. This instrument contains questions relating to the bed time, rise time, and duration of sleep episodes across the past month (Buysse et al., 1989). Additionally, to better characterize recent sleep status, participants further completed sleep logs $3 \mathrm{~d}$ before each experimental session.

Participants conformed to the structured sleep schedule during the month before the experiment, including across the $3 \mathrm{~d}$ before the experimental session. Specifically, in the month leading up to the study, participants reported average bed times before 1:00 A.M. (mean: 12:07 A.M., $\mathrm{SD} \pm 44 \mathrm{~min}$ ), rise times before 9:00 A.M. (mean: 8:25 A.M., SD \pm 41 $\mathrm{min}$ ), and sleep duration lengths between 7 and $9 \mathrm{~h}$ (mean: $7.63 \mathrm{~h}, \mathrm{SD} \pm$ $0.65 \mathrm{~h}$ ). Similarly, for the $2 \mathrm{~d}$ before the start of the structured sleep schedule, participants reported sleep-log mean bed times of 12:16 A.M. $(\mathrm{SD} \pm 40 \mathrm{~min})$, mean rise times of 8:05 A.M. (SD $\pm 43 \mathrm{~min})$, and mean sleep durations of $7.71 \mathrm{~h}(\mathrm{SD} \pm 0.62 \mathrm{~h}$ ). While it is important to note the inherent limitations of self-report measures, these findings support the likelihood that participants entered the study in a rested state, and that their normative schedules were congruent with the study requirements.

Changes in mood and anxiety state were assessed at 9:00 A.M. on the experimental mornings, in both rested and deprived conditions, using the Positive and Negative Affective Scale (Watson et al., 1988) and the State-Trait Anxiety Inventory (Spielberger et al., 1983), respectively. As alterations in mood and anxiety commonly co-occur with sleep disruption (Harvey et al., 2011), these measures provide an important test of whether changes in pain experience were not due to heightened anxiety and/or reduced mood.

Thermal sensitivity assessments. Quantitative sensory testing was performed within the scanner room, with the participant supine, by the same investigator in each session. Testing was performed on the left leg, and thermal stimuli were delivered using a $3 \times 3 \mathrm{~cm}$ contact thermode consisting of Peltier elements (TSA-II Neurosensory Analyzer, Medoc Advanced Medical System). In all following tests, cutoff temperature was set at $50^{\circ} \mathrm{C}$.

Warm detection thresholds were measured using the ascending method of limits (Gracely and Naliboff, 1996). A series of 6 ascending stimuli, starting at a baseline temperature of $32^{\circ} \mathrm{C}$ and increasing at a rate of $1^{\circ} \mathrm{C} / \mathrm{s}$, were delivered until the participant terminated the stimuli with a button press when warmth was detected, at which point the temperature returned to baseline at a rate of $2^{\circ} \mathrm{C} / \mathrm{s}$. The warm detection threshold measured before the first experimental session was used to determine the nonpainful warm stimulus later used in the fMRI task. Specifically, $2^{\circ} \mathrm{C}$ was added to each participant's baseline warm detection threshold, and this new temperature was presented to subjects to confirm that it was perceived as "warm, but not painful."

To determine the high temperature, painful stimulus for use in the fMRI task, baseline suprathreshold sensitivity, a temperature corresponding to a 7/10 (with 10 being unbearable pain) on a visual analog pain scale, was obtained for participants before their first session. A standard staircase psychophysics method was used in which the temperature is changed on a trial-by-trial basis according to the participant's rating (Gracely and Naliboff, 1996). In this method, a series of 8 temperatures was successively applied to accurately estimate a consistently painful temperature. Starting at the baseline temperature of $32^{\circ} \mathrm{C}$, stimuli were increased at a rate of $4^{\circ} \mathrm{C} / \mathrm{s}$ until the target temperature was reached and held for $10 \mathrm{~s}$. The first suprathreshold test stimulus was set at $45^{\circ} \mathrm{C}$ for all participants and adjusted based on subsequent individual responses. Following a ramp-down at a rate of $4^{\circ} \mathrm{C} / \mathrm{s}$, participants were asked to rate the intensity of the previous temperature on a visual analog pain scale. Unless the stimulus was rated as the target of 7 of 10, the subsequent trial temperature was adjusted by $1^{\circ} \mathrm{C}$ to bring it closer to the target. Once a temperature was identified as having the target intensity, stimuli were held constant for subsequent trials until it was rated as a 7/10 for 3 trials in a row, or the end of the stimuli set was reached, in which case, the last 3 temperatures were averaged and used as the individualized painful temperature in the fMRI task.

Thermal pain thresholds were measured in each experimental condition (sleep-rested, sleep-deprived) using the ascending method of limits. Here, participants are instructed to terminate the increasing temperature with a button press once the stimulus was perceived to be unpleasant, at which point the temperature returned to baseline at a rate of $4^{\circ} \mathrm{C} / \mathrm{s}$. The mean of these 6 temperatures was then used for subsequent analyses. In each of the two experimental sessions, pain thresholds were assessed 30 min before scanning.

$f M R I$ acquisition. BOLD contrast functional images were acquired with echo-planar $\mathrm{T} 2{ }^{\star}$-weighted (EPI) imaging using a Siemens 3 tesla MRI scanner with a 12-channel head coil. Each image volume consisted of 37 descending $3.5 \mathrm{~mm}$ slices $(96 \times 96$ matrix; TR $=2000 \mathrm{~ms}$; TE $=22$ $\mathrm{ms}$; size $3.5 \times 3.5 \times 3.0 \mathrm{~mm}$, flip angle $=50^{\circ}, 0.5 \mathrm{~mm}$ slice spacing). A high-resolution T1-weighted structural scan was acquired at the end of the sleep-rested session $(256 \times 256$ matrix; $\mathrm{TR}=1900$; TE $=2.52$; flip angle $=9^{\circ}$; FOV $256 \mathrm{~mm} ; 1 \times 1 \times 1 \mathrm{~mm}$ voxels).

$f M R I$ thermal pain sensitivity task. Each scanning session for the thermal pain sensitivity task consisted of a pseudo-randomly ordered sequence of two stimulus intensity phases: painful hot and nonpainful warm. Twelve painful and 12 nonpainful warm stimulus "trials" (24, total) were presented across three scanning blocks in each of the sleeprested and sleep-deprived conditions. Each block was separated by at least $60 \mathrm{~s}$, with each of the three blocks lasting $\sim 5 \mathrm{~min}$.

For each of the 24 experimental trials per session, participants were presented with a visual cue signaling impending thermal stimulation lasting $6 \mathrm{~s}$, after which the cue was removed from the screen and replaced with a fixation cross, followed by painful/nonpainful stimulation. The stimulation period consisted of a ramp-up period in which heat increased at a rate of $4^{\circ} \mathrm{C} / \mathrm{s}$ and was held at the target temperature for $12 \mathrm{~s}$. Consistent with prior fMRI studies of neural pain assessments (Becerra et al., 1999; Brown et al., 2011; Zunhammer et al., 2015), only the $12 \mathrm{~s}$ period of peak temperature stimulation was used in modeling of brain activity during painful/nonpainful trials. The heat returned to baseline $\left(32^{\circ} \mathrm{C}\right)$ at a rate of $8^{\circ} \mathrm{C} / \mathrm{s}$, after which an $8 \mathrm{~s}$ period of relief from stimulation occurred. Finally, after each stimulation trial, subjects rated both the intensity and unpleasantness of the previous temperature on an 11 point scale. All trial durations were jittered according to a uniform distribution, producing intertrial interval variability that is optimal for mixed block/event-related designs (Petersen and Dubis, 2012).

Online Mechanical Turk Study. The online phase of the study was conducted through MTurk. We sought to assess a cohort of at least 50 participants who reported experiencing some current pain, thereby allowing a determination of whether night-to-night changes in sleep predicted subsequent day-to-day changes in their pain experience. All participants were queried at 4 time points across 2 nights and days. In the 
mornings, participants reported on the prior nights' sleep, and in the evenings, reported on the days' pain.

A total of 236 (mean \pm SD age, $36.61 \pm 10.8$ years; $50 \%$ female) participants were surveyed. Only participants who reported pain on one or both assessment days, an IP address in the United States, and a prior online MTurk approval rating of $\geq 95 \%$ were included in subsequent analyses. Based on these factors, a subset of 60 participants (mean \pm SD age, $38.4 \pm 10.4$ years, $42 \%$ female) of candidates surveyed were eligible for participation (Fig. 1B).

Across the experimental phase, participants quantified a number of factors related to sleep and pain within specified time windows of the mornings and evenings. In morning assessments, participants filled out the Karolinska Sleep Diary, which included bed time, sleep latency, wake-up time, sleep duration, and subjective sleep quality on a 5 point scale (Akerstedt et al., 1994). Sleep efficiency was calculated from these daily diaries, based on percentage of time asleep out of total sleep duration (i.e., total sleep time minus sleep latency and total wake time after sleep onset). To characterize changes in affect, participants reported current mood and anxiety, using the Positive and Negative Affective Scale and State-Trait Anxiety Inventory questionnaires, respectively.

To assess the influence of nightly sleep on subsequent next-day pain, participants quantified their physical pain experience that day (input between 6:00 P.M., day's end) and rated the average magnitude of that pain on a 100 point scale (0 representing No Pain and 100 representing Worst Pain Imaginable).

Similar to the in-laboratory experiment, the online study used a within-subject, repeated-measures design. This minimized differences beyond the variable of sleep that could otherwise influence the outcome measure of pain (e.g., sex, age, health status). We deliberately did not provide instructions to subjects to curtail or elongate their sleep to maintain ecologically naturalistic sleep changes. To maximize the representativeness of the online sample, participants were deliberately not excluded based on age or health status, including the presence of chronic pain or pain disorders. Data points for either sleep or pain metrics that were $\pm 2 \mathrm{SD}$ of the group mean were considered outliers and excluded from analyses.

\section{Statistical analyses}

fMRI preprocessing. Preprocessing and data analysis were performed using the Statistical Parametric Mapping (SPM12, RRID:SCR_007037) software implemented in MATLAB R2015b (MATLAB, RRID: SCR_001622). Functional images were motion corrected and slice time corrected. Structural images were then coregistered to the mean functional image and normalized to a standard template. Functional images were spatially normalized to the MNI template using those parameters and smoothed using a $6 \mathrm{~mm}$ FWHM Gaussian kernel. For all preprocessing steps, we used the default parameters in SPM12. For each subject, trial-related activity was assessed by convolving a vector of trial onsets with a canonical hemodynamic response function. The six movementrelated covariates (three rigid-body translations and three rotations determined from the realignment preprocessing step) were used as regressors in the design matrix for modeling movement-related artifact in the time series. To further address the influence of motion on BOLD data, framewise displacement of head motion was calculated based on the motion parameters estimated during preprocessing using the ArtRepair toolbox (ArtRepair for robust fMRI, RRID:SCR_005990). TRs with framewise displacement values $>0.8$ were interpolated with the nearest artifact-free TRs surrounding the time point affected by motion. Autocorrelation in the data was accommodated for using a first-order autoregressive (AR1) model, estimated by pooling over suprathresholds (Friston et al., 2002).

To control for physiological noise, 5 principal components of CSF and white matter (WM) signal were added as regressors to the design matrix, implemented through the CompCor pipeline (Behzadi et al., 2007). Extraction of CSF/WM signal was derived from probabilistic maps segmented from T1-weighted structural images of each participant, using the segment function within SPM12. CSF/WM masks were then thresholded at a probability of 0.99 for WM and 0.95 for CSF, converted to functional-scan resolution, and eroded to eliminate isolated voxels and minimize partial volume effects.

fMRI analyses. A GLM was specified for each participant, using the task-related and nuisance regressors outlined above, to investigate the neural effects of interest (Friston et al., 1994). Contrasts were created at the first (individual) level with two events: painful and nonpainful heat. Specifically, the $12 \mathrm{~s}$ period during which the stimulus was at its peak temperature, excluding temperature ramp-up and ramp-down periods, was used to construct these contrasts. The experimental hypotheses were tested at the second (group) level using a priori ROIs centered on previously reported coordinates. Averaged activity from the current study was extracted from these

ROIs and further analyses with correction for multiple statistical tests were performed on these values. The approach prevents analysis circularity arising from nonindependence of the selected regions and the results and is in accord with standardized fMRI methods (Poldrack, 2007; Poldrack and Mumford, 2009).

A set of cortical and subcortical ROIs were defined on the basis of three convergent criteria. First, regions were selected from previously reported fMRI patterns associated with objective pain intensity. These regions were derived from machine-learning analysis of fMRI brain activity during thermal pain stimulation, and thus, represent a set of candidate neural regions whose activity reliably relates to objective noxious stimulus intensity (Wager et al., 2013). Within this network, only those regions in which increased activity was independently and significantly $(q<0.05$ false discovery rate $[\mathrm{FDR}]$ ) related to the painful stimuli were considered in the current ROI analysis.

Second, regions contributing to subjective pain experience, but not directly related to objective stimulus intensity, were considered. These regions were derived from prior findings of fMRI brain activity associated with subjective thermal pain experience (Woo et al., 2017). This was important in the context of the current experiment because prior studies of sleep loss have shown changes in subjective estimates of pain in response to the same objective noxious stimulus intensity (Tiede et al., 2010; Campbell et al., 2011). Here again, only those regions in which activity independently and significantly $(q<0.05)$ contributed to subjective pain were included as candidate ROIs.

Third, affective processes participate in painful experiences (Price, 2000; Wiech and Tracey, 2009), and this may contribute to the hyperalgesic effect of sleep deprivation. We therefore additionally required that a priori pain-related ROIs were known to be sleep loss-sensitive in prior studies of basic affective and emotional neural processing (Venkatraman et al., 2007; Yoo et al., 2007; Gujar et al., 2011; Goldstein et al., 2013; Greer et al., 2013; Goldstein-Piekarski et al., 2015).

From these three criteria, a set of 15 candidate ROIs emerge, reflecting the Venn diagram overlap of these previously published empirical studies: areas that are conjointly involved in objective noxious stimulus processing, subjective pain sensation, and sensitive to the effects of sleep deprivation in affective tasks. Sphere sizes of the ROIs used in the current study were selected to approximate the cluster sizes $\left(\mathrm{mm}^{3}\right)$ reported in the original studies from which coordinates were taken (Table 1).

Pain and brain analysis. To characterize the impact of sleep deprivation on the pain-related ROIs, average contrast estimates (Pain vs No Pain) were extracted from each ROI volume independently and condition differences were calculated using two-tailed paired $t$ tests. All statistical tests were performed within MATLAB. Consistent with imaging recommendations to correct for multiple statistical comparisons across the 15 ROIs, the Benjamini-Hochberg procedure for controlling FDR was applied, with $q=0.05$ (Benjamini and Hochberg, 1995). This procedure addresses the problem of simultaneously performing multiple significance tests by using an adaptive stepwise procedure to control the expected ratio of erroneous rejections to the number of total rejections. $p$ values that survived the FDR criterion after correction were considered statistically significant.

To test the relationships between sleep loss-related changes in ROI brain activity and subjective pain reports, percentage change in pain thresholds and suprathreshold pain (intensity and unpleasantness) were calculated for each subject, and Pearson correlated to changes in pain 
Table 1. ROI analysis ${ }^{a}$

\begin{tabular}{|c|c|c|c|c|c|c|}
\hline Region (L/R) & $\begin{array}{l}\text { Radius } \\
(\mathrm{mm})\end{array}$ & $x$ & $y$ & $z$ & $p$ & $r$ \\
\hline \multicolumn{7}{|c|}{ Sleep deprivation > sleep-rested } \\
\hline Somatosensory (R) & 8 & 36 & -45 & 59 & 0.004 & 0.55 \\
\hline Dorsal ACC (R) & 8 & 16 & -7 & 39 & 0.02 & 0.04 \\
\hline Superior parietal (R) & 5 & 26 & -42 & 66 & 0.51 & -0.04 \\
\hline \multicolumn{7}{|c|}{ Sleep-rested $>$ sleep-deprived } \\
\hline Middle insula $(\mathrm{R})^{b}$ & 8 & 32 & 4 & 11 & 0.0005 & -0.02 \\
\hline Thalamus $(\mathrm{L})^{b}$ & 4 & -10 & 6 & 10 & 0.005 & -0.54 \\
\hline Anterior insula $(\mathrm{L})^{b}$ & 8 & -27 & 25 & 0 & 0.005 & 0.24 \\
\hline$N A c C^{b}$ & 6 & \pm 9 & 2 & -7 & 0.005 & 0.23 \\
\hline Operculum (R) & 5 & 57 & 1 & 5 & 0.04 & -0.36 \\
\hline Substantia nigra (R) & 4 & 10 & -8 & 12 & 0.09 & -0.31 \\
\hline Caudate & 4 & \pm 18 & -2 & 24 & 0.38 & -0.32 \\
\hline IFG (R) & 4 & 64 & 24 & -6 & 0.47 & -0.47 \\
\hline Middle cingulate/SMA & 10 & 6 & -6 & 48 & 0.48 & 0.01 \\
\hline Precuneus (R) & 4 & 4 & -54 & 48 & 0.60 & 0.32 \\
\hline $\mathrm{ACC}(\mathrm{R})$ & 6 & 12 & 28 & 28 & 0.63 & 0.36 \\
\hline DLPFC (R) & 8 & 33 & 35 & 42 & 0.90 & 0.10 \\
\hline
\end{tabular}

${ }^{a}$ Brain ROI analysis showing radius, $\mathrm{MNI}$ coordinates, and $p$ and $r$ values of extracted spheres. $r$, Pearson correlations between pain-related brain changes (sleep-deprived vs sleep-rested) and changes in thermal pain threshold (sleeprested vs sleep-deprived). ACC, Anterior cingulate cortex; IFG, inferior frontal gyrus; SMA, supplementary motor area; DLPFC, dorsolateral prefrontal cortex.

${ }^{b}$ Significant, following FDR correction, for paired comparison (sleep-rested $\langle/\rangle$ sleep-deprived) of pain-related brain activity.

ROIs. The same FDR approach described above was used to correct for multiple tests.

Mechanical Turk analysis. Day-to-day (day 2 to day 1) changes in reported pain and night-to-night (night 2 to night 1 ) changes in sleep were calculated for each participant. Testing the hypothesis that natural fluctuations in three measures of sleep predicted subsequent next-day pain, participants were categorically separated on the basis of whether they experienced an increase or decrease from one night to the next in each of the three sleep measures $(>0 \% /<0 \%$ change). Of the 60 participants, 55\% improved their sleep quality from one night to the next. Forty-percent of subjects improved their sleep quantity/duration, and $52 \%$ improved their sleep efficiency relative to the first assessed night. Daily changes in reported pain were compared between categorical groups, defined on the basis of each of the three measured sleep factors, using two-tailed paired t tests $(\alpha=0.05)$.

\section{Results}

Sleep deprivation and pain sensitivity

In-laboratory study. Consistent with the hypothesis, the standardized thermal pain threshold assessment (performed outside of the MRI scanner) demonstrated a significant impact of sleep deprivation on pain processing. Specifically, sleep loss expanded the temperature range for classifying a stimulus as painful through a lowering of pain threshold, such that sleep-deprived participants registered pain at a significantly lower temperature than when sleep-rested $\left(t_{(24)}=4.36, p=0.0002\right.$; Fig. 2$)$.

Inside of the scanner, there were no significant main effects of sleep deprivation on ratings of intensity and unpleasantness of suprathreshold noxious blocks (intensity: $t_{(24)}=0.67, p=0.51$; unpleasantness: $\left.t_{(24)}=0.94, p=0.36\right)$. This was similarly true for ratings of nonpainful stimulus blocks (warmth) (both: $t_{(24)}<$ $0.98, p>0.33)$. Such a result importantly demonstrates that sleep loss did not significantly alter sensation of unambiguous nonnoxious thermal stimuli.

Therefore, sleep deprivation expressly resulted in a shift, specifically a lowering, in the decision threshold to classify a stimulus as painful (assessed outside of the scanner), as shown by the significant decrease in pain thresholds, whereas the extremes of pain sensitivity used in the canonical scanning paradigm re-

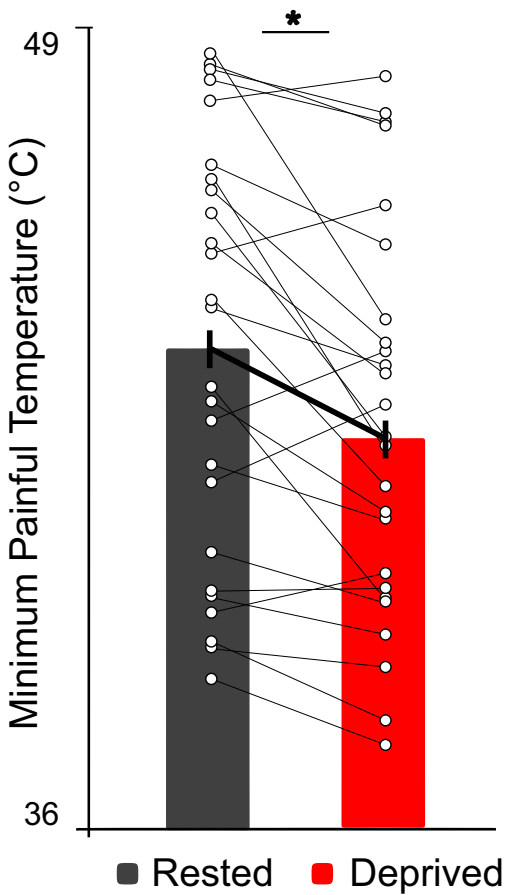

Figure 2. Thermal pain threshold. Following sleep deprivation, there was an increase in pain sensitivity demonstrated by a significant lowering of pain thresholds, relative to sleep-rested. Sleep-deprived mean $\pm S D=42.47 \pm 3.22^{\circ} \mathrm{C}$; Sleep-rested mean $\pm S D=43.89 \pm 3.49^{\circ} \mathrm{C}$. ${ }^{*} p<0.0002$.

mained similar in each condition. The latter meant that any differences in brain activity between the sleep-rested and sleep deprivation conditions assessed during fMRI scanning could not simply be accounted for by one condition having an imbalance in subjective experience ratings.

\section{Sleep deprivation and neural pain processing}

Analyses of fMRI data revealed bidirectional changes in brain activity in participants under conditions of sleep loss within recognized a priori pain regions, relative to when sleep-rested. Specifically, sleep deprivation significantly increased pain reactivity within right primary somatosensory cortex $\left(t_{(24)}=3.20, p=\right.$ 0.004; FDR-corrected for multiple ROI tests at $q=0.05$; Fig. 3) (Benjamini and Hochberg, 1995). This finding is consistent both with the role of somatosensory cortex in the registration and representation of noxious painful stimuli (including heat), and the contralateral crossing of sensory signals because the stimulation was to the left side of the body (Coghill et al., 1999; Craig, 2002; Apkarian et al., 2005; Atlas et al., 2014).

In contrast to the somatosensory cortex, significant decreases in activity were observed within subcortical a priori ROIs of the thalamus as well as the NAcc following sleep deprivation, relative to the rested condition (thalamus: $t_{(24)}=-3.13, p=0.005$; NAcc: $t_{(24)}=-3.11, p=0.005$; FDR-corrected). Such NAcc disengagement is congruent with the prediction of a shift in decision threshold regarding what constitutes a painful stimuli because this striatal region is associated with pain valuation and relief (Altier and Stewart, 1999; Baliki et al., 2010; Navratilova and Porreca, 2014; Lee et al., 2015; Woo et al., 2015, 2017; Sardi et al., 2018). Appropriate cortical estimation of pain within the human brain is not limited to representations within primary somatosensory strip but involves further higher-order cortical evaluation within interoceptive integration regions of the insula 
A

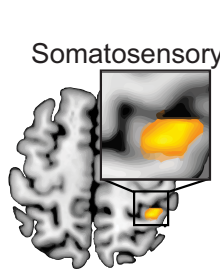

- Rested

- Deprived
B

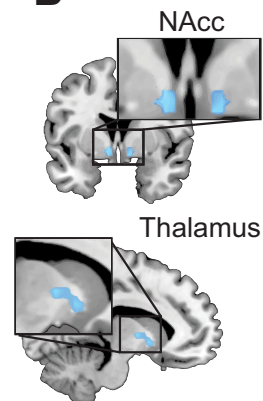

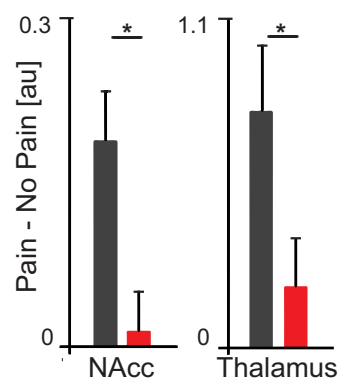
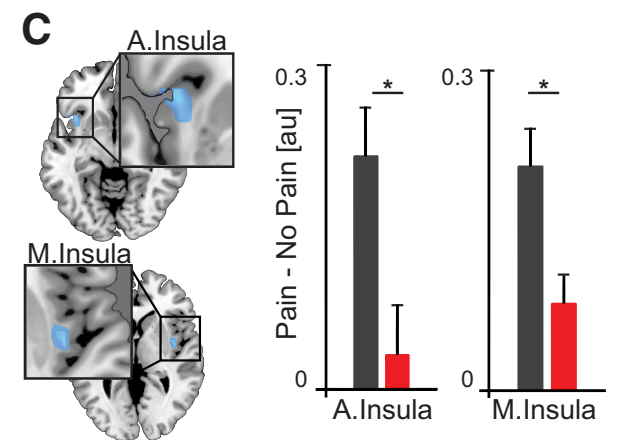

Figure 3. Changes in pain-related brain activity. Data are mean \pm SD. $\boldsymbol{A}-\boldsymbol{C}$, Brain maps (left) displaying pain-related R0ls that were significantly altered by sleep deprivation, relative to sleep-rested (all FDR-corrected). Bar plots represent brain activity (Pain $>$ No Pain) contrast estimates. Somatosensory: sleep-deprived mean $=0.09 \pm 0.34$; sleep-rested mean $=-0.34 \pm 0.46$. NAcc: sleep-deprived mean $=0.02 \pm 0.18$; sleep-rested mean $=0.19 \pm 0.21$. Thalamus: sleep-deprived mean $=-0.21 \pm 0.81$; sleep-rested mean $=0.79 \pm 1.06$. Anterior insula: sleep-deprived mean $=0.12 \pm 0.82$; sleep-rested mean $=0.79 \pm 0.8$. Middle insula: sleep-deprived mean $=0.08 \pm 0.12$; sleep-rested mean $=0.21 \pm 0.17$. Error bars indicate SEM. ${ }^{*} p<$ 0.005. Nucleus Accumbens (NAcc); Anterior and middle insula (A. Insula and M. Insula, respectively). Threshold for display set to $p<0.005$ (whole-brain, uncorrected).

and cingulate (Craig, 2003; Critchley et al., 2004). A priori ROI regions of the right middle insula and left anterior insula demonstrated significant decreases in activity during pain under conditions of sleep deprivation (right middle insula: $t_{(24)}=-4.06, p=0.0005$; left anterior insula: $t_{(24)}=-3.11, p=0.005$; FDRcorrected). The latter, left hemisphere association is relevant considering that left anterior insula receives parasympathetic afferent information, and lesions of this insula region increase pain sensitivity, indicative of a loss of anodyne homeostatic signaling under sleep deprivation conditions (Craig, 2005; Starr et al., 2009).

No other regions within the a priori cortical pain network demonstrated significance that exceeded FDR correction for multiple ROIs, including the cingulate cortex. Several were significant below this threshold and reported for completeness in Table 1, but not discussed further.

Having identified condition differences (sleep-rested $<>$ sleep-deprived) in pain-related brain activity, we next sought to determine whether the extent of change in activity within these pain-related regions predicted interindividual differences in the change in pain threshold caused by sleep deprivation.

Across participants, the extent of amplified somatosensory pain reactivity positively and significantly predicted the lowering of pain thresholds (i.e., the expansion of pain sensation) caused by sleep deprivation $\left(r_{(23)}=0.55, p=0.004\right.$; FDR-corrected; Fig. $4 A$ ). In addition, the degree of decrease in thalamic activity associated with sleep deprivation significantly and negatively predicted the lowering of pain thresholds across individuals $\left(r_{(23)}=\right.$ $-0.54, p=0.005$; FDR-corrected; Fig. $4 B$ ). That is, the extent of increased somatosensory pain reactivity, and the associated decrease in thalamic reactivity, following sleep deprivation, both predicted greater pain-sensitivity increases across individuals following sleep loss. No other such associations (positive or negative) were identified within other a priori ROIs (all $p>0.05$; Table 1).

Therefore, the somatosensory cortex and left thalamus both showed a main effect of sleep deprivation; furthermore, their respective extent of change in pain reactivity predicted the magnitude of expanded sensitivity to pain caused by sleep deprivation, measured using the threshold assessment.

Mood and anxiety are known to be impacted by sleep deprivation (Harvey et al., 2011) and, independent of sleep, are recognized to influence pain (Wiech and Tracey, 2009). To test the specificity of the pain-amplifying effect of sleep deprivation, relationships between self-reported changes in mood and anxiety following sleep deprivation were examined in relation to the change in pain.

Levels of both positive and negative mood, reported immediately before scanning, were numerically, but not significantly, different between sleep-deprived and sleep-rested conditions (positive mood: sleep-deprived mean $=17.4 \pm 4.8 \mathrm{SD}$; sleeprested mean $=20.12 \pm 7.4 ; p=0.14$; negative mood: sleepdeprived mean $=15.2 \pm 1.9 \mathrm{SD}$; sleep-rested mean $=16.64 \pm$ 4.8; $p=0.19)$. Importantly, however, these sleep loss-related differences in both positive and negative mood did not significantly correlate with the change in pain thresholds (positive mood: $r_{(23)}=-0.04, p=0.85$; negative mood: $r_{(23)}=-0.18, p=$ $0.39)$. There were similar numeric but nonsignificant differences in state anxiety following sleep deprivation. However, like mood, these sleep loss-associated changes in self-reported anxiety were also not correlated with changes in pain thresholds measured at the same time (anxiety: sleep-deprived mean $=35.5 \pm 10.2 \mathrm{SD}$; 
- Increased - Decreased

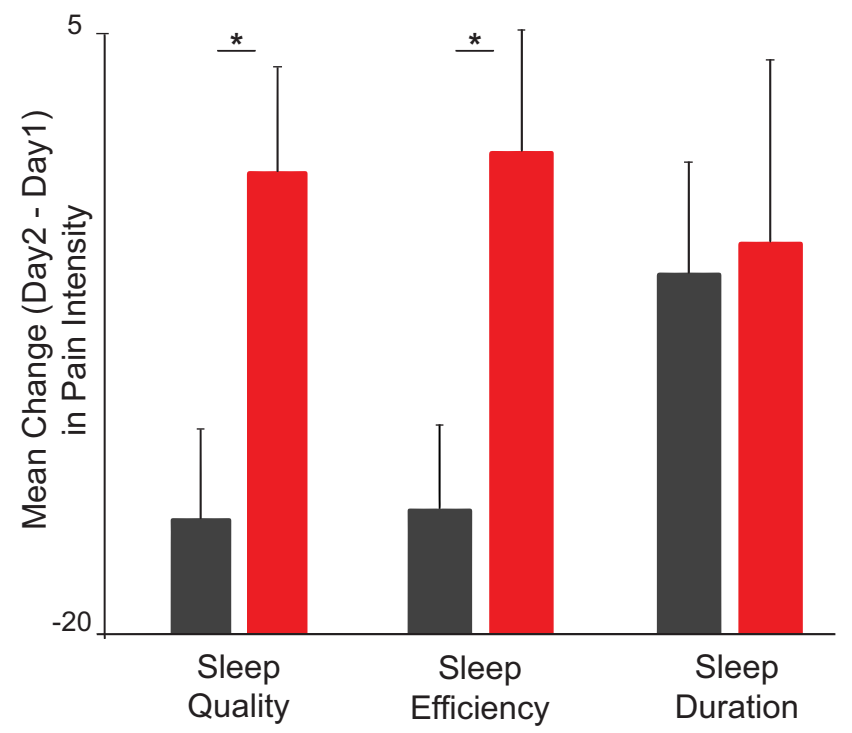

Figure 5. Online study results. Data are mean $\pm S D$. Bar graph represents participants experiencing a decrease (red bar) or increase (black) in measures of sleep efficiency (SE) (Night 2 to Night 1 mean decreased SE $=-3.8 \pm 5.6 \%$; Night 2 to Night 1 mean increased SE $=$ $10.1 \pm 11.1 \%$ ), quality (SQ) (Night 2 to Night 1 mean decreased SQ $=-0.48 \pm 0.75$; Night 2 to Night 1 mean increased SQ $=1.7 \pm 0.81$ ), and duration (Night 2 to Night 1 mean decreased duration $=-54.375 \pm 45.7 \mathrm{~min}$; Night 2 to Night 1 mean increased duration $=90.7 \pm 48.9$ min). Error bars indicate SEM. Each point represents a single-subject's change in reported pain from day 1 to day 2. Night-to-night decreases in sleep efficiency and quality, but not duration, resulted in corresponding higher pain from one day to the next, relative to those with night-tonight increases in these sleep measures. ${ }^{*} p<0.05$.

sleep-rested mean $=32 \pm 9.3 \mathrm{SD} ; p=0.18 ; r_{(23)}=-0.27, p=$ $0.19)$.

Together, this suggests that the observed associations between sleep and pain were not parsimoniously accounted for by cooccurring changes in affect in the current study.

\section{Ecological night-to-night changes in sleep and subsequent day-to-day changes in pain}

While the in-laboratory experiment establishes that acute total sleep deprivation leads to altered neural pain processing and an associated expansion of sensitivity to pain, it intimates, but does not itself address, whether ecologically relevant night-to-night variation in sleep, within an individual, similarly results in consequential day-to-day changes in experienced pain during the subsequent day. This was the goal of the online experiment.

Supporting the experimental hypothesis, and the directional predictions from the in-laboratory acute sleep deprivation experiment, participants who had a decrease in sleep efficiency from one night to the next experienced a corresponding increase in pain from one day to the next $\left(t_{(58)}=2.4, p=0.018\right.$; Fig. 5; each bar represents a different group of subjects).

A similar relationship was observed for night-to-night changes in subjective sleep quality, such that reduction in the quality of sleep was associated with increased consequential daily pain $\left(t_{(58)}=2.1, p=0.04\right.$; Fig. 5). Interestingly, however, nightly changes in total amount of sleep did not similarly predict daily changes in pain $\left(t_{(58)}=0.39, p=0.7\right)$.

Although the current study was not designed to prove a causal order of changes in sleep and changes in pain, the data suggest that altered sleep statistically forecasts next-day pain. To further examine whether this was true, we used partial correlation anal- ysis to examine the relative strength of the association between nightly sleep quality and next-day pain while controlling for effects of pain on the previous day. If sleep quality still predicted next-day pain, even when controlling for prior pain status, it would lend support to the idea that it is the change in sleep that is robustly predicting subsequent changes in pain. Additionally, we extend this test and performed a second partial correlation analysis of pain and next-day sleep quality while controlling for prior sleep status. This affords an additional exploration that speaks to causal precedence; were pain to be associated with subsequent sleep quality independent of the effects of prior sleep, it would suggest that pain changes precede sleep changes, not vice versa.

When controlling for the effects of pain intensity on day 1 , a significant relationship between sleep quality and pain on the following day (day 2) still remained $(r=-0.26, p=0.04)$. This suggests that the evening sleep quality is a stronger predictor of subsequent next-day pain than prior pain predicting subsequent sleep. In addition, when controlling for prior sleep quality (night 1 ), the partial correlation between pain and sleep quality on the following night (night 2$)$ was nonsignificant $(r=-0.04, p=$ 0.72 ). These results therefore affirm that it is sleep quality that predicts next-day pain, even when factoring in the influence of prior pain status.

Importantly, self-reported changes in mood and anxiety were not associated with day-to-day fluctuations in experienced pain (positive mood: $r_{(58)}=-0.14, p=0.29$; negative mood: $r_{(58)}=$ $-0.21, p=0.11$; anxiety: $\left.r_{(58)}=0.07, p=0.59\right)$. As with the in-laboratory study, this would indicate that the observed associations between sleep and pain do not appear to be robustly explained by parallel changes in these emotional constructs.

Thus, ecological night-to-night variability in sleep efficiency and subjective quality within an individual, but not quantity, predicted consequential day-to-day reductions in corresponding pain. This online counterpart to the in-laboratory experiment corroborates the pain-enhancing effect of inadequate sleep but further establishes that even modest changes in sleep quality are sufficient to affect pain and can be observed within individuals across nights, beyond cross-sectional examinations.

\section{Discussion}

Together, these findings help establish the following: (1) that acute sleep deprivation expands the temperature range for classifying a stimulus as painful, specifically through a lowering of pain thresholds; (2) that the underlying neural correlates of such sleep loss-induced hyperalgesia involved increasing pain reactivity within primary somatosensory cortex, yet a blunting of pain reactivity in higher-order valuation analgesic-relief, and decisionmaking regions of the striatum and insula cortex; and (3) that the same effect can be observed following more ecologically relevant changes in sleep, such that modest night-to-night changes in sleep quality within an individual (increases and decreases) determine consequential day-to-day changes in experienced pain (decreases and increases, respectively).

\section{Sleep and neural pain processing}

Current models view pain as a homeostatic emotion. Pain is constructed from ascending interoceptive afferents conveying viscerosensory and viscerochemical changes in body state. These signals project to registration sites within brainstem, and on to subcortical and cortical sensory, homeostatic regulatory, and valuation centers. Pain is therefore a specific sensation and a felt emotion, one that drives homeostatic behavior (Craig, 2002, 2003; Critchley and Harrison, 2013). 
Fitting this distributed model of pain processing, our findings describe a bidirectional set of neural changes within the recognized network of the brain that processes pain following sleep deprivation. Specifically, sleep loss triggered an increase in pain reactivity within sensory-discriminative regions of somatosensory cortex. Notably, heightened somatosensory cortex reactivity was lateralized to the opposite side of the cortex, relative to the application of the noxious stimulus on the body. This is fitting with the contralateral organization of primary somatosensation and indicates an intensification of first-order cortical pain registration resulting from sleep deprivation (Craig, 2002).

Beyond this main condition effect, the magnitude of increased activity in somatosensory cortex following sleep deprivation predicted the relative decrease in thermal pain threshold in the sleepdeprived state, the greater the increase in somatosensory cortex noxious reactivity, the greater the shift (lowering) of pain sensitivity across individuals. That the somatosensory cortex explained individual differences in pain thresholds aligns well with the role of somatosensory cortex in the initial magnitude registration of pain (Coghill et al., 1999; Apkarian et al., 2005; Atlas et al., 2014). Therefore, one component of the exacerbation of pain caused by a lack of sleep involves amplified primary pain processing in rudimentary areas of human sensory cortex, the activity of which scales with sleep loss-induced expansion of pain sensation.

A converse decrease in pain reactivity was observed in subcortical NAcc, thalamus, and insula cortex. A recognized function of the insula involves the integration of afferent signals from somatosensory cortex into second-order representation of affective body state, available for conscious perception (Craig, 2002, 2003; Critchley and Harrison, 2013). Indeed, the broad connectivity of the insula with regions, including the prefrontal and orbitofrontal cortices, thalamus, ACC, and NAcc, suggests that the insula is well situated to be a key regulator of sensed pain (Craig, 2002, 2003).

Related, the NAcc is linked with pain valuation, decisionmaking, and analgesia-related pain-regulation in a number of studies (Navratilova and Porreca, 2014; Woo et al., 2015, 2017). This includes NAcc-associated relief from ongoing pain, the analgesic influence of which is transacted through descending pain efferent pathways (Altier and Stewart, 1999; Baliki et al., 2010; Lee et al., 2015; Woo et al., 2015, 2017; Sardi et al., 2018). In addition, the NAcc has reciprocal connections to pain-integrating and pain-modulating cortical regions, including ventromedial prefrontal cortex, ACC, insula, and somatosensory cortex (Borsook et al., 2010).

The thalamus is consistently activated in acute pain experiments and plays a role in the gating of contralateral ascending sensory information as it reaches the cortex (Craig, 2003). However, the function of the thalamus in pain processing is not limited to classical sensory gating. First, decreases in thalamic gray matter structure and functional activity are a characteristic of multiple chronic pain conditions with different etiologies (Pridmore et al., 2003; Apkarian et al., 2004; Gustin et al., 2011). Indeed, the reduction of thalamic function due to lesions predicts the severity of central pain conditions, including reduced pain thresholds (Vartiainen et al., 2016), the proposed mechanism of which is the loss, or disinhibition, of normal thalamocortical pain signaling (Craig et al., 1996; Craig, 2000; Vartiainen et al., 2016).

Building on this functional circuitry, increased somatosensory, yet reduced insula, thalamus, and NAcc, pain reactivity challenges any rudimentary model in which sleep loss triggers hyperalgesia through unidirectional increases in neural pain representations. Instead, the impact of insufficient sleep on pain involves both an amplification of primary cortical pain registration, potentially due to thalamic disinhibition, and a shift in affective valuation and decision-making involving insula and NAcc. We propose that these changes can parsimoniously account for the observed shift in the decision threshold for categorizing a potentially noxious stimulus as painful through misrepresentation of pain signals within insula and NAcc. This misrepresentation of the salience of thermal stimuli may consequentially lead to an inaccurate amplification (gain increase) of pain registration within somatosensory cortex. Indeed, this exact pattern has been reported in patients with lesions to the insula cortex, who demonstrate simultaneously higher pain sensitivity, elevated somatosensory cortex activity, and an absence of painmodulatory activity in the insula (Starr et al., 2009).

\section{Ecological variations in sleep and pain}

Further testing our hypothesis, the second study demonstrated that subtle, ecological changes in sleep in the general population predict consequential day-to-day changes in self-reported pain intensity. Interestingly, it was night-to-night changes in sleep quality, rather than sleep quantity, that were deterministic of changes in pain sensitivity.

Insufficient sleep, self-reported, has been shown to correlate, cross-sectionally, with self-reported pain in a sample of the general population (Smith and Haythornthwaite, 2004; Afolalu et al., 2018) and clinical cohorts (Tang et al., 2012). Our findings add to this link by establishing that, beyond cross-sectional associations, changes in the quality of night-to-night sleep within an individual in a micro-longitudinal assay, more so than the quantity, predict consequential day-to-day changes in pain intensity (Edwards et al., 2008). That even modest reductions in sleep quantity/quality impact next-day pain is increasingly relevant given the continued erosion of sleep time in developed nations (National Sleep Foundation Sleep in America Poll, 2013; http://sleepfoundation.org/sleep-polls-data/ sleep-in-america-poll/2013-exercise-and-sleep). This is additionally pertinent when considered alongside the escalating prevalence and economic health burden of pain in these same countries (Goldberg and McGee, 2011). Indeed, findings from a recent national poll (National Sleep Foundation Sleep in America Poll, 2015; https:// sleepfoundation.org/sleep-polls-data/sleep-in-america-poll/2015sleep-and-pain) demonstrate that $64 \%$ of those suffering chronic pain, and $54 \%$ of those with acute pain, report co-occurring poor sleep quality.

Our findings perhaps offer encouragement, in that they suggest that even modest improvements in sleep quality have the potential to reduce subjectively significant pain. That is, sleep can be seen as a modifiable risk factor and intervention target for pain, and the magnitude of sleep improvement necessary to result in measurable reductions in pain is modest, and thus pragmatically realistic.

Given that pain is among the costliest health burdens in both direct healthcare spending and indirect lost productivity (Phillips, 2009), improving sleep may additionally confer broader economic savings. This concern (and therapeutic opportunity) is exemplified by the inpatient hospital setting, where sleep quality is consistently poor and pain is frequently co-occurring. Placing sleep quality closer to the center of inpatient treatment approaches may reduce patient suffering, and potentially, the dosage of narcotic and non-narcotic analgesics (Walker, 2018).

\section{Study considerations}

Potential limitations of the current studies should be noted. First, the in-laboratory experiment was performed with healthy young 
adults. Because pain becomes more frequent in middle and late life, it remains to be determined whether these findings translate across the lifespan. Second, only noxious heat was examined in the laboratory study. It therefore remains unclear whether different brain changes would be observed using different stimulus modalities, such as noxious mechanical, electrical, or chemical stimuli. This seems less likely, however, considering that previous studies show a commonality of pain-enhancing effects of sleep loss across such modalities (Lautenbacher et al., 2006). Third, as brain activity was only collected during the suprathreshold pain sensitivity task, inferences regarding the mechanisms of reduced thresholds rely on significant correlations with subsequent suprathreshold neural pain reactivity. Finally, while post hoc analyses established that changes in nightly sleep statistically forecast next-day pain changes, even when accounting for prior pain status, they do not prove this fact causally (as this experiment was not designed to directly address causal ordering). Nevertheless, a meta-analysis of longitudinal and micro-longitudinal studies in both clinical and nonclinical populations has established that two-thirds of relevant studies demonstrate stronger evidence supporting a causal influence of prior sleep on subsequent experienced pain, rather than the opposite direction (Finan et al., 2013).

\section{References}

Afolalu EF, Ramlee F, Tang NK (2018) Effects of sleep changes on painrelated health outcomes in the general population: a systematic review of longitudinal studies with exploratory meta-analysis. Sleep Med Rev 39: 82-97.

Akerstedt T, Hume K, Minors D, Waterhouse J (1994) The subjective meaning of good sleep, an intraindividual approach using the Karolinska Sleep Diary. Percept Mot Skills 79:287-296.

Altier N, Stewart J (1999) The role of dopamine in the nucleus accumbens in analgesia. Life Sci 65:2269-2287.

Apkarian AV, Sosa Y, Sonty S, Levy RM, Harden RN, Parrish TB, Gitelman DR (2004) Chronic back pain is associated with decreased prefrontal and thalamic gray matter density. J Neurosci 24:10410-10415.

Apkarian AV, Bushnell MC, Treede RD, Zubieta JK (2005) Human brain mechanisms of pain perception and regulation in health and disease. Eur J Pain 9:463-484.

Atlas LY, Lindquist MA, Bolger N, Wager TD (2014) Brain mediators of the effects of noxious heat on pain. Pain 155:1632-1648.

Baliki MN, Geha PY, Fields HL, Apkarian AV (2010) Predicting value of pain and analgesia: nucleus accumbens response to noxious stimuli changes in the presence of chronic pain. Neuron 66:149-160.

Becerra LR, Breiter HC, Stojanovic M, Fishman S, Edwards A, Comite AR, Gonzalez RG, Borsook D (1999) Human brain activation under controlled thermal stimulation and habituation to noxious heat: an fMRI study. Magn Reson Med 41:1044-1057.

Behzadi Y, Restom K, Liau J, Liu TT (2007) A component based noise correction method (CompCor) for BOLD and perfusion based fMRI. Neuroimage 37:90-101.

Benjamini Y, Hochberg Y (1995) Controlling the false discovery rate: a practical and powerful approach to multiple testing. J R Stat Soc B 57:289300.

Borsook D, Upadhyay J, Chudler EH, Becerra L (2010) A key role of the basal ganglia in pain and analgesia: insights gained through human functional imaging. Mol Pain 6:27.

Brown JE, Chatterjee N, Younger J, Mackey S (2011) Towards a physiologybased measure of pain: patterns of human brain activity distinguish painful from non-painful thermal stimulation. PLoS One 6:e24124.

Buysse DJ, Reynolds CF, Monk TH, Berman SR, Kupfer DJ (1989) The Pittsburgh Sleep Quality Index: a new instrument for psychiatric practice and research. Psychiatry Res 28:193-213.

Campbell CM, Bounds SC, Simango MB, Witmer KR, Campbell JN, Edwards RR, Haythornthwaite JA, Smith MT (2011) Self-reported sleep duration associated with distraction analgesia, hyperemia, and secondary hyperalgesia in the heat-capsaicin nociceptive model. Eur J Pain 15:561-567.

Coghill RC, Sang CN, Maisog JM, Iadarola MJ (1999) Pain intensity pro- cessing within the human brain: a bilateral, distributed mechanism. J Neurophysiol 82:1934-1943.

Craig A (2000) The functional anatomy of lamina I and its role in poststroke central pain. In: Progress in brain research, pp 137-151. Amsterdam: Elsevier.

Craig AD (2002) How do you feel? Interoception: the sense of the physiological condition of the body. Nat Rev Neurosci 3:655-666.

Craig AD (2003) A new view of pain as a homeostatic emotion. Trends Neurosci 26:303-307.

Craig AD (2005) Forebrain emotional asymmetry: a neuroanatomical basis? Trends Cogn Sci 9:566-571.

Craig AD, Reiman EM, Evans A, Bushnell MC (1996) Functional imaging of an illusion of pain. Nature 384:258-260.

Critchley HD, Harrison NA (2013) Visceral influences on brain and behavior. Neuron 77:624-638.

Critchley HD, Wiens S, Rotshtein P, Ohman A, Dolan RJ (2004) Neural systems supporting interoceptive awareness. Nat Neurosci 7:189-195.

Edwards RR, Almeida DM, Klick B, Haythornthwaite JA, Smith MT (2008) Duration of sleep contributes to next-day pain report in the general population. Pain 137:202-207.

Faraut B, Léger D, Medkour T, Dubois A, Bayon V, Chennaoui M, Perrot S (2015) Napping reverses increased pain sensitivity due to sleep restriction. PLoS One 10:e0117425.

Finan PH, Goodin BR, Smith MT (2013) The association of sleep and pain: an update and a path forward. J Pain 14:1539-1552.

Friston KJ, Holmes AP, Worsley KJ, Poline JP, Frith CD, Frackowiak RS (1994) Statistical parametric maps in functional imaging: a general linear approach. Hum Brain Mapp 2:189-210.

Friston KJ, Glaser DE, Henson RN, Kiebel S, Phillips C, Ashburner J (2002) Classical and Bayesian inference in neuroimaging: applications. Neuroimage 16:484-512.

Goldberg DS, McGee SJ (2011) Pain as a global public health priority. BMC Public Health 11:770.

Goldstein AN, Greer SM, Saletin JM, Harvey AG, Nitschke JB, Walker MP (2013) Tired and apprehensive: anxiety amplifies the impact of sleep loss on aversive brain anticipation. J Neurosci 33:10607-10615.

Goldstein-Piekarski AN, Greer SM, Saletin JM, Walker MP (2015) Sleep deprivation impairs the human central and peripheral nervous system discrimination of social threat. J Neurosci 35:10135-10145.

Gracely RH, Naliboff BD (1996) Measurement of pain sensation. In: Pain and touch, Ed 2 (Kruger L, ed), pp 244-308. San Diego: Academic.

Greer SM, Goldstein AN, Walker MP (2013) The impact of sleep deprivation on food desire in the human brain. Nat Commun 4:2259.

Gujar N, Yoo SS, Hu P, Walker MP (2011) Sleep deprivation amplifies reactivity of brain reward networks, biasing the appraisal of positive emotional experiences. J Neurosci 31:4466-4474.

Gustin SM, Peck CC, Wilcox SL, Nash PG, Murray GM, Henderson LA (2011) Different pain, different brain: thalamic anatomy in neuropathic and non-neuropathic chronic pain syndromes. J Neurosci 31:5956-5964.

Harvey AG, Murray G, Chandler RA, Soehner A (2011) Sleep disturbance as transdiagnostic: consideration of neurobiological mechanisms. Clin Psychol Rev 31:225-235.

Lautenbacher S, Kundermann B, Krieg JC (2006) Sleep deprivation and pain perception. Sleep Med Rev 10:357-369.

Lee M, Manders TR, Eberle SE, Su C, D'amour J, Yang R, Lin HY, Deisseroth K, Froemke RC, Wang J (2015) Activation of corticostriatal circuitry relieves chronic neuropathic pain. J Neurosci 35:5247-5259.

Lentz MJ, Landis CA, Rothermel J, Shaver JL (1999) Effects of selective slow-wave sleep disruption on musculoskeletal pain and fatigue in middle aged women. J Rheumatol 26:1586-1592.

Navratilova E, Porreca F (2014) Reward and motivation in pain and pain relief. Nat Neurosci 17:1304-1312.

Petersen SE, Dubis JW (2012) The mixed block/event-related design. Neuroimage 62:1177-1184.

Phillips CJ (2009) The cost and burden of chronic pain. Rev Pain 3:2-5.

Poldrack RA (2007) Region of interest analysis for fMRI. Soc Cogn Affect Neurosci 2:67-70.

Poldrack RA, Mumford JA (2009) Independence in ROI analysis: where is the voodoo? Soc Cogn Affect Neurosci 4:208-213.

Price DD (2000) Psychological and neural mechanisms of the affective dimension of pain. Science 288:1769-1772. 
Pridmore S, Samilowitz H, Oberoi G (2003) The brain and chronic pain. German J Psychiatry 6:8-15.

Redgrave P, Gurney K, Reynolds J (2008) What is reinforced by phasic dopamine signals? Brain Res Rev 58:322-339.

Roehrs T, Hyde M, Blaisdell B, Greenwald M, Roth T (2006) Sleep loss and REM sleep loss are hyperalgesic. Sleep 29:145-151.

Sardi NF, Tobaldini G, Morais RN, Fischer L (2018) Nucleus accumbens mediates the pronociceptive effect of sleep deprivation: the role of adenosine A2A and dopamine D2 receptors. Pain 159:75-84.

Schrimpf M, Liegl G, Boeckle M, Leitner A, Geisler P, Pieh C (2015) The effect of sleep deprivation on pain perception in healthy subjects: a metaanalysis. Sleep Med 16:1313-1320.

Schuh-Hofer S, Wodarski R, Pfau DB, Caspani O, Magerl W, Kennedy JD, Treede RD (2013) One night of total sleep deprivation promotes a state of generalized hyperalgesia: a surrogate pain model to study the relationship of insomnia and pain. Pain 154:1613-1621.

Schultz W (2013) Updating dopamine reward signals. Curr Opin Neurobiol 23:229-238.

Smith MT, Haythornthwaite JA (2004) How do sleep disturbance and chronic pain inter-relate? Insights from the longitudinal and cognitivebehavioral clinical trials literature. Sleep Med Rev 8:119-132.

Spielberger CD, Gorsuch RL, Lushene R, (1983) Manual for the state-trait anxiety inventory. Palo Alto, CA: Consulting Psychologists Press.

Starr CJ, Sawaki L, Wittenberg GF, Burdette JH, Oshiro Y, Quevedo AS, Coghill RC (2009) Roles of the insular cortex in the modulation of pain: insights from brain lesions. J Neurosci 29:2684-2694.

Tang NK, Goodchild CE, Sanborn AN, Howard J, Salkovskis PM (2012) Deciphering the temporal link between pain and sleep in a heterogeneous chronic pain patient sample: a multilevel daily process study. Sleep 35:675-687A

Tiede W, Magerl W, Baumgärtner U, Durrer B, Ehlert U, Treede RD (2010) Sleep restriction attenuates amplitudes and attentional modulation of pain-related evoked potentials, but augments pain ratings in healthy volunteers. Pain 148:36-42.
Timmermann L, Ploner M, Haucke K, Schmitz F, Baltissen R, Schnitzler A (2001) Differential coding of pain intensity in the human primary and secondary somatosensory cortex. J Neurophysiol 86:1499-1503.

Tracey I, Mantyh PW (2007) The cerebral signature for pain perception and its modulation. Neuron 55:377-391.

Vartiainen N, Perchet C, Magnin M, Creac'h C, Convers P, Nighoghossian N, Mauguière F, Peyron R, Garcia-Larrea L (2016) Thalamic pain: anatomical and physiological indices of prediction. Brain 139:708-722.

Venkatraman V, Chuah YM, Huettel SA, Chee MW (2007) Sleep deprivation elevates expectation of gains and attenuates response to losses following risky decisions. Sleep 30:603-609.

Wager TD, Atlas LY, Lindquist MA, Roy M, Woo CW, Kross E (2013) An fMRI-based neurologic signature of physical pain. N Engl J Med 368:1388-1397.

Walker MP (2018) A sleep prescription for medicine. The Lancet 391:2598-2599.

Watson D, Clark LA, Tellegen A (1988) Development and validation of brief measures of positive and negative affect: the PANAS scales. J Pers Soc Psychol 54:1063-1070.

Wiech K, Tracey I (2009) The influence of negative emotions on pain: behavioral effects and neural mechanisms. Neuroimage 47:987-994.

Woo CW, Roy M, Buhle JT, Wager TD (2015) Distinct brain systems mediate the effects of nociceptive input and self-regulation on pain. PLoS Biol 13:e1002036

Woo CW, Schmidt L, Krishnan A, Jepma M, Roy M, Lindquist MA, Atlas LY, Wager TD (2017) Quantifying cerebral contributions to pain beyond nociception. Nat Commun 8:14211.

Yoo SS, Gujar N, Hu P, Jolesz FA, Walker MP (2007) The human emotional brain without sleep: a prefrontal amygdala disconnect. Curr Biol 17:R877-R878.

Zunhammer M, Geis S, Busch V, Greenlee MW, Eichhammer P (2015) Effects of intranasal oxytocin on thermal pain in healthy men: a randomized functional magnetic resonance imaging study. Psychosom Med 77:156166. 